\title{
Analytical Study of Fractional-Order Multiple Chaotic FitzHugh-Nagumo Neurons Model Using Multistep Generalized Differential Transform Method
}

\author{
Shaher Momani, ${ }^{1,2}$ Asad Freihat, ${ }^{3}$ and Mohammed AL-Smadi ${ }^{4}$ \\ ${ }^{1}$ Department of Mathematics, Faculty of Science, University of Jordan, Amman 11942, Jordan \\ ${ }^{2}$ Nonlinear Analysis and Applied Mathematics (NAAM) Research Group, Faculty of Science, King Abdulaziz University, \\ Jeddah 21589, Saudi Arabia \\ ${ }^{3}$ Pioneer Center for Gifted Students, Ministry of Education, Jerash 26110, Jordan \\ ${ }^{4}$ Applied Science Department, Ajloun College, Al-Balqa Applied University, Ajloun 26816, Jordan
}

Correspondence should be addressed to Shaher Momani; s.momani@ju.edu.jo

Received 8 March 2014; Accepted 13 May 2014; Published 12 June 2014

Academic Editor: Dumitru Baleanu

Copyright (C) 2014 Shaher Momani et al. This is an open access article distributed under the Creative Commons Attribution License, which permits unrestricted use, distribution, and reproduction in any medium, provided the original work is properly cited.

\begin{abstract}
The multistep generalized differential transform method is applied to solve the fractional-order multiple chaotic FitzHugh-Nagumo (FHN) neurons model. The algorithm is illustrated by studying the dynamics of three coupled chaotic FHN neurons equations with different gap junctions under external electrical stimulation. The fractional derivatives are described in the Caputo sense. Furthermore, we present figurative comparisons between the proposed scheme and the classical fourth-order Runge-Kutta method to demonstrate the accuracy and applicability of this method. The graphical results reveal that only few terms are required to deduce the approximate solutions which are found to be accurate and efficient.
\end{abstract}

\section{Introduction}

Mathematical modeling method of real-life phenomena is widely applied in medicine and biology. Specifically, the understanding of neural system model plays an important role in several branches of medical science and technology such as neuroscience, brain activity, chemical reaction kinetics, and behavior of cardiac tissue [1-6]. So it has attracted many medical researchers over the last two decades in order to understand the biogenesis, mechanism, and function. Despite that the formulation of such systems is considerably simple, the lacking understanding of their complex behaviors remains to be a very challenging task, especially when the results are expected in a very short time. However, the advent of computers significant progress has been made recently to reduce this gap.

Furthermore, the FHN neural system is one of the best mathematical models describing the electrical activity in the field of electrocardiology, which is a simplified model for the qualitative characteristics and dynamical and neuronal investigations of electrical propagation in the myocardium. For a comprehensive introduction in this field, we refer to [7-16].

In this paper, the chaotic FHN neurons model under external electrical stimulation is given by the following three coupled equations with different gap junctions:

$$
\begin{aligned}
\frac{d x_{1}}{d t}= & x_{1}\left(x_{1}-1\right)\left(1-r x_{1}\right)-y_{1}-\breve{g}_{12}\left(x_{1}-x_{2}\right) \\
& -\breve{g}_{13}\left(x_{1}-x_{3}\right)+\left(\frac{a}{\omega}\right) \cos \omega t+d_{1}, \\
\frac{d y_{1}}{d t}= & b x_{1}, \\
\frac{d x_{2}}{d t}= & x_{2}\left(x_{2}-1\right)\left(1-r x_{2}\right)-y_{2}-\breve{g}_{12}\left(x_{2}-x_{1}\right) \\
& -\breve{g}_{23}\left(x_{2}-x_{3}\right)+\left(\frac{a}{\omega}\right) \cos \omega t+d_{2},
\end{aligned}
$$




$$
\begin{aligned}
& \frac{d y_{2}}{d t}=b x_{2} \\
& \frac{d x_{3}}{d t}=x_{3}\left(x_{3}-1\right)\left(1-r x_{3}\right)-y_{3}-\breve{g}_{13}\left(x_{3}-x_{1}\right) \\
& -\breve{g}_{23}\left(x_{3}-x_{2}\right)+\left(\frac{a}{\omega}\right) \cos \omega t+d_{3}, \\
& \frac{d y_{3}}{d t}=b x_{3}
\end{aligned}
$$

where $x$ and $y$ represent the state variables of a neuron representing the activation potential and the recovery voltage, respectively; $\left(x_{1}, y_{1}\right),\left(x_{2}, y_{2}\right)$, and $\left(x_{3}, y_{3}\right)$ represent the states of the master, the first slave, and the second slave FHN neuron, respectively; $\breve{g}_{12}, \breve{g}_{13}$, and $\breve{g}_{23}$ represent the strengths of gap junctions between the master and the first slave neurons, between the master and the second slave neurons, and between the two slave neurons, respectively. Disturbances at the master, the first slave, and the second slave neurons are represented by $d_{1}, d_{2}$, and $d_{3}$, respectively. The term $(a / \omega) \cos \omega t$ represents the external stimulation current with time $t$ and angular frequency $\omega$. Here, we use the angular frequency $\omega$ and the amplitude $a$ as dimensionless quantities as specified for FHN neurons model.

The literature on this subject is quite vast, for example, the full FitzHugh model on an infinite domain has been studied in [17]. In [18], the Hopf bifurcations have analyzed FHN model for nerve conduction. The dynamics of uncertain coupled chaotic delayed FHN neurons with various parametric variations under external electrical stimulation have been investigated in [19], where separate conditions for single-input and multiple-input control schemes for synchronization of a wide class of FHN systems were provided. In [20], the authors have discussed the synchronization of three coupled chaotic FHN neurons under external electrical stimulation with different gap junctions. Moreover, numerical simulation of the FHN equations has been presented using the variational iteration method and Adomian decomposition method [21]. Whilst, the analytical solutions for the FHN model in the case where a collection of unstable cells is surrounded by a collection of stable cells have been generated in [22].

Nowadays, fractional calculus has been used to model physical and engineering processes, which are found to be best described by fractional differential equations [23-26]. It is worth noting that the standard mathematical models of integer-order derivatives, including nonlinear models, do not work adequately in many cases. More recently, fractional calculus has become a powerful tool to describe the dynamics of chaotic neurons system, which appear frequently in many branches of medical science. Chaotic neurons systems have a profound effect on its approximate solutions and are highly sensitive to time step sizes. Thus, it will be beneficial to find a reliable analytical tool to test its long-term accuracy and efficiency. The multistep generalized differential transform method (MSGDTM) is powerful in investigating approximate solutions of various kinds of these systems.
In this paper, the attention is given to obtain the approximate solution of the fractional-order multiple chaotic FHN neurons model under external electrical stimulation with different gap junctions using the MSGDTM. This method is only a simple modification of the generalized differential transform method (GDTM), in which it is treated as an algorithm in a sequence of small intervals (i.e., time step) for finding accurate approximate solutions to the corresponding systems. The approximate solutions obtained by using the GDTM are valid only for a short time. The ones obtained by using the MSGDTM are more valid and accurate during a long time and are in good agreement with the classical Runge-Kutta method numerical solution when the order of the derivative is one.

The remainder of this paper is organized as follows. In next section, we present basic facts, definitions, and notations related to the fractional calculus and MSGDTM. In Section 3, the MSGDTM is applied to the fractional-order multiple chaotic FHN neurons model. In Section 4, numerical simulation is shown graphically to illustrate the feasibility and effectiveness of the proposed method. Finally, the conclusions are drawn in Section 5.

\section{The Multistep Generalized Differential Transform Method (MSGDTM)}

To describe the MSGDTM [26-29], we consider the following initial value problem for systems of fractional differential equations:

$$
D_{*}^{\alpha_{i}} y_{i}(t)=f_{1}\left(t, y_{1}, y_{2}, \ldots, y_{n}\right), \quad i=1,2, \ldots, n,
$$

subject to the initial conditions

$$
y_{i}\left(t_{0}\right)=c_{i}, \quad i=1,2, \ldots, n,
$$

where $D_{*}^{\alpha_{i}}$ is the Caputo fractional derivative of order $\alpha_{i}$, and $0<\alpha_{i} \leqslant 1$, for $i=1,2, \ldots, n$, and the Caputo fractional derivative of $f(x)$ of order $\alpha>0$ with $a \geq 0$ is defined as

$$
\left(D_{a}^{\alpha} f\right)(x)=\frac{1}{\Gamma(m-\alpha)} \int_{a}^{x} \frac{f^{(m)}(t)}{(x-t)^{\alpha+1-m}} d t,
$$

for $m-1<\alpha \leq m, m \in \mathbb{N}, x \geq a, f \in C_{-1}^{m}$. For more details about the fractional calculus theory, see [30-32].

Let $\left[t_{0}, T\right]$ be the interval over which we want to find the solution of the initial value problem (2)-(3). The differential transform of the $k$ th-order derivative of a function $f(t)$ on a subinterval $\left[t_{m-1}, t_{m}\right]$ is defined as follows:

$$
F(k)=\frac{1}{\Gamma(k+1)}\left[\frac{d^{k} f(t)}{d t^{k}}\right]_{t=t_{m}} .
$$

Using (5), one can easily prove the following corollary.

Corollary 1. If $f(t)=\sin (\omega t)$, then $F(k)=\left(\omega^{k} / \Gamma(k+1)\right)$ $\sin \left(\omega t_{m}+\pi k / 2\right)$, while if $f(t)=\cos (\omega t)$, then $F(k)=\left(\omega^{k} / \Gamma(k+\right.$ 1)) $\cos \left(\omega t_{m}+\pi k / 2\right)$. 
In actual applications of GDTM, the Kth-order approximate solution of the initial value problem (2)-(3) can be expressed by the finite series

$$
y_{i}(t)=\sum_{i=0}^{K} Y_{i}(k)\left(t-t_{0}\right)^{k \alpha_{i}}, \quad t \in[0, T],
$$

where $Y_{i}(k)$ satisfied the recurrence relation

$$
\begin{array}{r}
\frac{\Gamma\left((k+1) \alpha_{i}+1\right)}{\Gamma\left(k \alpha_{i}+1\right)} Y_{i}(k+1)=F_{i}\left(k, Y_{1}, Y_{2}, \ldots, Y_{n}\right), \\
i=1,2, \ldots, n,
\end{array}
$$

and $Y_{i}(0)=c_{i}$ and $F_{i}\left(k, Y_{1}, Y_{2}, \ldots, Y_{n}\right)$ are the differential transform of function $f_{i}\left(t, y_{1}, y_{2}, \ldots, y_{n}\right)$ for $i=1,2, \ldots, n$.

Assume that the interval $\left[t_{0}, T\right]$ is divided into $M$ subintervals $\left[t_{m-1}, t_{m}\right], m=1,2, \ldots, M$, of equal step size $h=$ $\left(T-t_{0}\right) / M$ by using the nodes $t_{m}=t_{0}+m h$. The main ideas of the MSGDTM are as follows. Firstly, we will apply the GDTM to the initial value problem (2)-(3) over the interval $\left[t_{0}, t_{1}\right]$. Then, we will obtain the approximate solution $y_{i, 1}(t)$, $t \in\left[t_{0}, t_{1}\right]$, using the initial condition $y_{i}\left(t_{0}\right)=c_{i}$, for $i=$ $1,2, \ldots, n$. For $m \geq 2$ and at each subinterval $\left[t_{m-1}, t_{m}\right]$, we will use the initial condition $y_{i, m}\left(t_{m-1}\right)=y_{i, m-1}\left(t_{m-1}\right)$ and apply the GDTM to the initial value problem (2)-(3) over the interval $\left[t_{m-1}, t_{m}\right]$. The process is repeated and generates a sequence of approximate solutions $y_{i, m}(t), m=1,2, \ldots, M$, for $i=1,2, \ldots, n$. Finally, the MSGDTM assumes the following solution:

$$
y_{i}(t)=\sum_{m=1}^{n} \chi_{v} y_{i, m}(t), \quad i=1,2, \ldots, n, m=1,2, \ldots, M,
$$

where

$$
\chi_{v}= \begin{cases}1, & t \in\left[t_{m-1}, t_{m}\right], \\ 0, & t \notin\left[t_{m-1}, t_{m}\right] .\end{cases}
$$

The new algorithm of the MSGDTM is simple for computational performance for all values of $t$. As we will see in the next section, the main advantage of the new algorithm is that the obtained solution converges for wide time regions.

\section{Applications of the MSGDTM for the Fractional-Order Multiple Chaotic FHN Neurons Model}

To demonstrate the applicability, accuracy, and efficiency of the MSGDTM for solving linear and nonlinear fractionalorder equations, we applied this scheme to the fractionalorder model of three coupled chaotic FHN neurons with different gap junctions [20], which is the lowest-order chaotic system among all the chaotic systems. Where the integerorder derivatives are replaced by the fractional-order derivatives as follows:

$$
\begin{aligned}
D_{*}^{\alpha_{1}} & x_{1}(t) \\
= & x_{1}\left(x_{1}-1\right)\left(1-r x_{1}\right)-y_{1}-\breve{g}_{12}\left(x_{1}-x_{2}\right) \\
& -\breve{g}_{13}\left(x_{1}-x_{3}\right)+\left(\frac{a}{w}\right) \cos (w t)+0.02 \sin (t),
\end{aligned}
$$

$$
\begin{aligned}
& D_{*}^{\alpha_{2}} y_{1}(t)=b x_{1}, \\
& D_{*}^{\alpha_{3}} x_{2}(t) \\
& =x_{2}\left(x_{2}-1\right)\left(1-r x_{2}\right)-y_{2}-\breve{g}_{12}\left(x_{2}-x_{1}\right) \\
& -\breve{g}_{23}\left(x_{2}-x_{3}\right)+\left(\frac{a}{w}\right) \cos (w t)+0.02 \sin (1.1 t) \\
& D_{*}^{\alpha_{4}} y_{2}(t)=b x_{2}, \\
& D_{*}^{\alpha_{5}} x_{3}(t) \\
& =x_{3}\left(x_{3}-1\right)\left(1-r x_{3}\right)-y_{3}-\breve{g}_{13}\left(x_{3}-x_{1}\right) \\
& -\breve{g}_{23}\left(x_{3}-x_{2}\right)+\left(\frac{a}{w}\right) \cos (w t)+0.02 \sin (1.2 t) \\
& D_{*}^{\alpha_{6}} y_{3}(t)=b x_{3},
\end{aligned}
$$

where $\breve{g}_{12}, \breve{g}_{13}$, and $\breve{g}_{23}$ are the strengths of gap junctions between the master and the first slave neurons, between the master and the second slave neurons, and between the two slave neurons, respectively; $r, a$, and $b$ are the system parameters and $x$ and $y$ are the state variables of a neuron representing the activation potential and the recovery voltage, respectively; $\left(x_{1}, y_{1}\right),\left(x_{2}, y_{2}\right)$, and $\left(x_{3}, y_{3}\right)$ are the states of the master, the first slave, and the second slave FHN neuron, respectively; and $\alpha_{i}, i=1,2,3,4,5,6$ are parameters describing the order of the fractional time-derivatives in the Caputo sense.

By applying the MSGDT algorithm to obtain the numerical solution for the fractional-order multiple chaotic FHN neurons model, the system (10) gives

$$
\begin{aligned}
& X_{1}(k+1) \\
& =\Gamma_{\alpha_{1}}\left(\sum_{l=0}^{k} X_{1}(l) X_{1}(k-l)-X_{1}(\kappa)-Y_{1}(k)\right. \\
& -r\left(-\sum_{l=0}^{k} X_{1}(l) X_{1}(k-l)\right. \\
& \left.+\sum_{j=0}^{k} \sum_{l=0}^{j} X_{1}(l) X_{1}(j-l) X_{1}(k-j)\right) \\
& -\breve{g}_{12}\left(X_{1}(\kappa)-X_{2}(\kappa)\right) \\
& -\breve{g}_{13}\left(X_{1}(\kappa)-X_{3}(\kappa)\right) \\
& +\frac{a(w)^{k-1}}{k !} \cos \left(\left(\frac{T}{M}\right) m+\frac{\pi k}{2}\right) \\
& \left.+\frac{0.02}{k !} \sin \left(\left(\frac{T}{M}\right) m+\frac{\pi k}{2}\right)\right), \\
& Y_{1}(k+1)=b \Gamma_{\alpha_{2}} X_{1}(\kappa),
\end{aligned}
$$



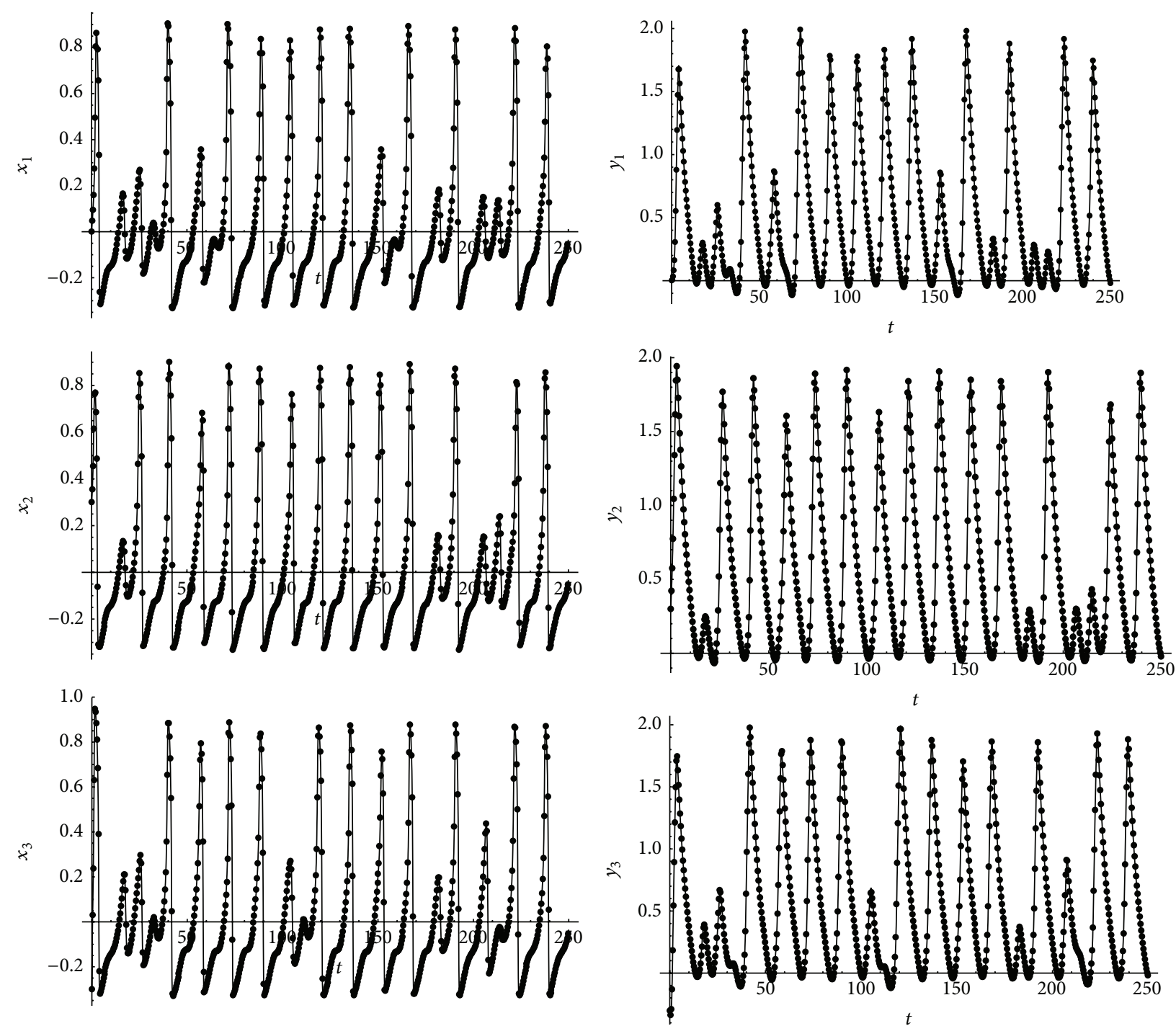

FIGURE 1: Numerical solutions of the FHN system; MSGDTM: dotted line; RK4: solid line, with $\alpha_{1}=\alpha_{2}=\alpha_{3}=\alpha_{4}=\alpha_{5}=\alpha_{6}=1$.

$$
\begin{array}{cc}
X_{2}(k+1) & Y_{2}(k+1)=b \Gamma_{\alpha_{4}} X_{2}(\kappa), \\
=\Gamma_{\alpha_{3}}\left(\sum_{l=0}^{k} X_{2}(i) X_{2}(k-l)-X_{2}(\kappa)-Y_{2}(k)\right. & X_{3}(k+1) \\
-r\left(-\sum_{l=0}^{k} X_{2}(l) X_{2}(k-l)\right. & =\Gamma_{\alpha_{5}}\left(\sum_{l=0}^{k} X_{3}(l) X_{3}(k-l)-X_{3}(\kappa)-Y_{3}(k)\right. \\
\left.+\sum_{j=0}^{k} \sum_{l=0}^{j} X_{2}(l) X_{2}(j-l) X_{2}(k-j)\right) & -r\left(-\sum_{l=0}^{k} X_{3}(l) X_{3}(k-l)\right. \\
-\breve{g}_{12}\left(X_{2}(\kappa)-X_{3}(\kappa)\right) & \left.+\sum_{j=0}^{k} \sum_{i=0}^{j} X_{3}(l) X_{3}(j-l) X_{3}(k-j)\right) \\
-\breve{g}_{23}\left(X_{2}(\kappa)-X_{3}(\kappa)\right) & -\breve{g}_{13}\left(X_{3}(\kappa)-X_{1}(\kappa)\right) \\
+\frac{a(w)^{k-1}}{k !} \cos \left(\left(\frac{T}{M}\right) m+\frac{\pi k}{2}\right) & -\breve{g}_{23}\left(X_{3}(\kappa)-X_{2}(\kappa)\right)
\end{array}
$$



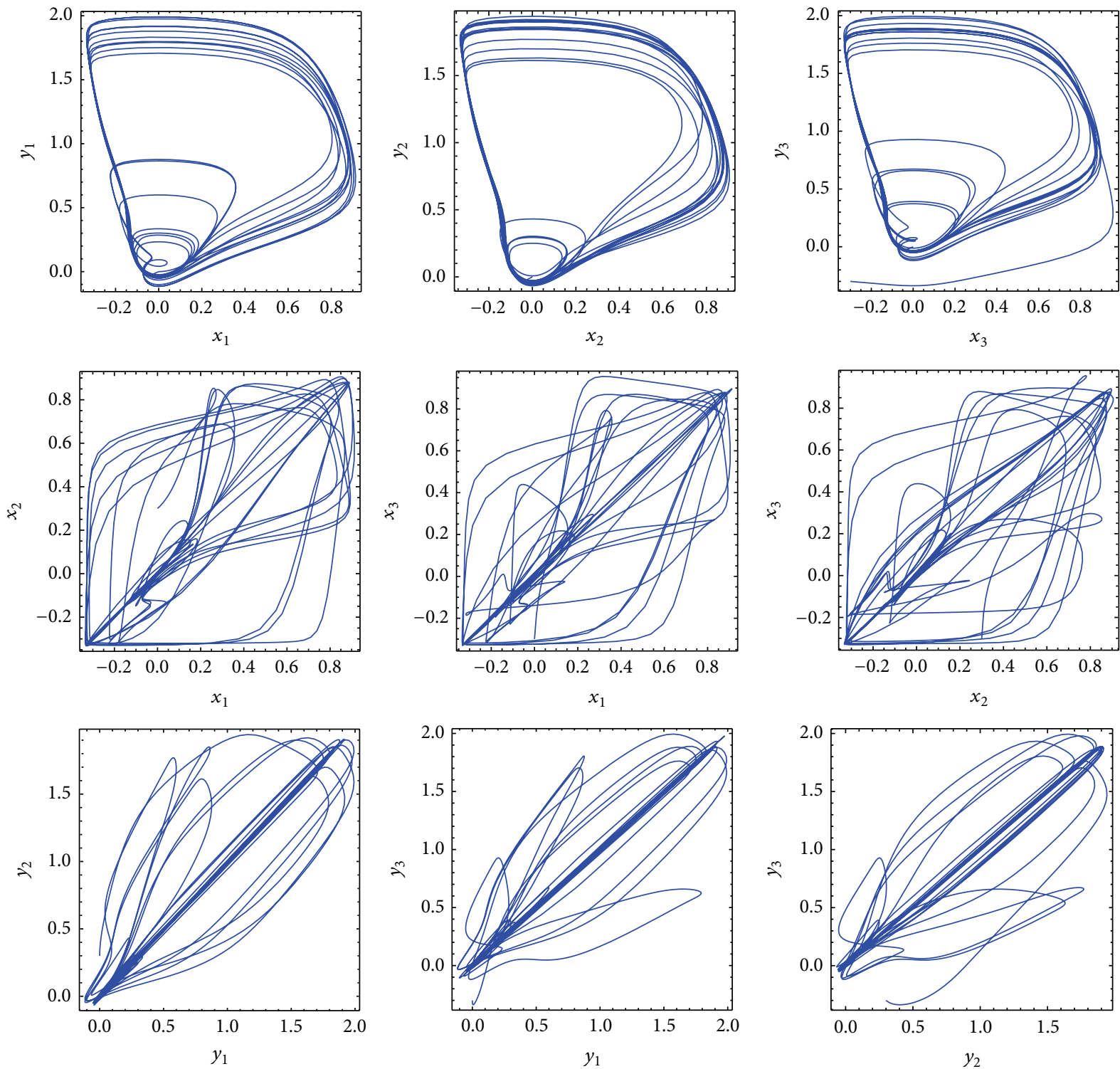

FIGURE 2: Phase plot of chaotic behavior of chaotic FHN neuronsis, with $\alpha_{1}=\alpha_{2}=\alpha_{3}=\alpha_{4}=\alpha_{5}=\alpha_{6}=1$.

$$
\begin{aligned}
& +\frac{a(w)^{k-1}}{k !} \cos \left(\left(\frac{T}{M}\right) m+\frac{\pi k}{2}\right) \\
& \left.+\frac{0.02(1.2)^{k}}{k !} \sin \left(\left(\frac{T}{M}\right) m+\frac{\pi k}{2}\right)\right),
\end{aligned}
$$

$Y_{3}(k+1)=b \Gamma_{\alpha_{6}} X_{3}(\kappa)$,

where $\Gamma_{\alpha_{i}}=\Gamma\left(\alpha_{i} k+1\right) / \Gamma\left(\alpha_{i}(k+1)+1\right), i=1,2, \ldots, 6, X_{i}(k)$ and and $Y_{i}(k)$ are the differential transformation of $x_{i}(t)$ and $y_{i}(t), i=1,2,3$, respectively. The differential transform of the initial conditions are given by $X_{1}(0)=c_{1}, Y_{1}(0)=c_{2}$, $X_{2}(0)=c_{3}, Y_{2}(0)=c_{4}, X_{3}(0)=c_{5}$, and $Y_{3}(0)=c_{6}$. In view of the differential inverse transform, the differential transform series solution for the system (10) can be obtained as

$$
\begin{aligned}
& x_{1}(t)=\sum_{n=0}^{N} X_{1}(n) t^{\alpha_{1} n}, \\
& y_{1}(t)=\sum_{n=0}^{N} Y_{1}(n) t^{\alpha_{2} n}, \\
& x_{2}(t)=\sum_{n=0}^{N} X_{2}(n) t^{\alpha_{3} n}, \\
& y_{2}(t)=\sum_{n=0}^{N} Y_{2}(n) t^{\alpha_{4} n}, \\
& x_{3}(t)=\sum_{n=0}^{N} X_{3}(n) t^{\alpha_{5} n} \\
& y_{3}(t)=\sum_{n=0}^{N} Y_{3}(n) t^{\alpha_{6} n} .
\end{aligned}
$$


According to the MSGDTM, the series solution for the system (10) is suggested by

$$
\begin{aligned}
& x_{1}(t)= \begin{cases}\sum_{n=0}^{K} X_{1,1}(n) t^{\alpha_{1} n}, & t \in\left[0, t_{1}\right], \\
\sum_{n=0}^{K} X_{1,2}(n)\left(t-t_{1}\right)^{\alpha_{1} n}, & t \in\left[t_{1}, t_{2}\right], \\
\vdots & \\
\sum_{n=0}^{K} X_{1, M}(n)\left(t-t_{M-1}\right)^{\alpha_{1} n}, & t \in\left[t_{M-1}, t_{M}\right],\end{cases} \\
& y_{1}(t)= \begin{cases}\sum_{n=0}^{K} Y_{1,1}(n) t^{\alpha_{2} n}, & t \in\left[0, t_{1}\right], \\
\sum_{n=0}^{K} Y_{1,2}(n)\left(t-t_{1}\right)^{\alpha_{2} n}, & t \in\left[t_{1}, t_{2}\right], \\
\vdots & \\
\sum_{n=0}^{K} Y_{1, M}(n)\left(t-t_{M-1}\right)^{\alpha_{2} n}, & t \in\left[t_{M-1}, t_{M}\right],\end{cases} \\
& x_{2}(t)= \begin{cases}\sum_{n=0}^{K} X_{2,1}(n) t^{\alpha_{3} n}, & t \in\left[0, t_{1}\right], \\
\sum_{n=0}^{K} X_{2,2}(n)\left(t-t_{1}\right)^{\alpha_{3} n}, & t \in\left[t_{1}, t_{2}\right], \\
\vdots & \\
\sum_{n=0}^{K} X_{2, M}(n)\left(t-t_{M-1}\right)^{\alpha_{3} n}, & t \in\left[t_{M-1}, t_{M}\right],\end{cases} \\
& y_{2}(t)= \begin{cases}\sum_{n=0}^{K} Y_{2,1}(n) t^{\alpha_{4} n}, & t \in\left[0, t_{1}\right], \\
\sum_{n=0}^{K} Y_{2,2}(n)\left(t-t_{1}\right)^{\alpha_{4} n}, & t \in\left[t_{1}, t_{2}\right], \\
\vdots & \\
\sum_{n=0}^{K} Y_{2, M}(n)\left(t-t_{M-1}\right)^{\alpha_{4} n}, & t \in\left[t_{M-1}, t_{M}\right],\end{cases} \\
& x_{3}(t)= \begin{cases}\sum_{n=0}^{K} X_{3,1}(n) t^{\alpha_{5} n}, & t \in\left[0, t_{1}\right], \\
\sum_{n=0}^{K} X_{3,2}(n)\left(t-t_{1}\right)^{\alpha_{5} n}, & t \in\left[t_{1}, t_{2}\right], \\
\vdots & \\
\sum_{n=0}^{K} X_{3, M}(n)\left(t-t_{M-1}\right)^{\alpha_{5} n}, & t \in\left[t_{M-1}, t_{M}\right],\end{cases} \\
& y_{3}(t)= \begin{cases}\sum_{n=0}^{K} Y_{3,1}(n) t^{\alpha_{6} n}, & t \in\left[0, t_{1}\right], \\
\sum_{n=0}^{K} Y_{3,2}(n)\left(t-t_{1}\right)^{\alpha_{6} n}, & t \in\left[t_{1}, t_{2}\right], \\
\vdots & \\
\sum_{n=0}^{K} Y_{3, M}(n)\left(t-t_{M-1}\right)^{\alpha_{6} n}, & t \in\left[t_{M-1}, t_{M}\right],\end{cases}
\end{aligned}
$$

where $X_{1, i}(n), Y_{1, i}(n), X_{2, i}(n), Y_{2, i}(n), X_{3, i}(n)$, and $Y_{2, i}(n)$, for $i=1,2, \ldots, M$, satisfy the following recurrence relations:

$$
\begin{aligned}
& X_{1, i}(k+1) \\
& =\Gamma_{\alpha_{1}}\left(\sum_{l=0}^{k} X_{1, i}(l) X_{1, i}(k-l)-X_{1, i}(\kappa)-Y_{1}(k)\right. \\
& -r\left(-\sum_{i=0}^{k} X_{1, i}(l) X_{1, i}(k-l)\right. \\
& \left.+\sum_{j=0}^{k} \sum_{l=0}^{j} X_{1, i}(l) X_{1, i}(j-l) X_{1, i}(k-j)\right) \\
& -\breve{g}_{12}\left(X_{1, i}(\kappa)-X_{2, i}(\kappa)\right) \\
& -\breve{g}_{13}\left(X_{1, i}(\kappa)-X_{3, i}(\kappa)\right) \\
& +\left(\frac{a}{w}\right) \frac{(w)^{k}}{k !} \cos \left(\left(\frac{T}{M}\right) m+\frac{\pi k}{2}\right) \\
& \left.+\frac{0.02}{k !} \sin \left(\left(\frac{T}{M}\right) m+\frac{\pi k}{2}\right)\right) \text {, } \\
& Y_{1, i}(k+1)=b \Gamma_{\alpha_{2}} X_{1, i}(\kappa) \text {, } \\
& X_{2, i}(k+1) \\
& =\Gamma_{\alpha_{3}}\left(\sum_{l=0}^{k} X_{2, i}(l) X_{2, i}(k-l)-X_{2, i}(\kappa)-Y_{2, i}(k)\right. \\
& -r\left(-\sum_{l=0}^{k} X_{2, i}(l) X_{2, i}(k-l)\right. \\
& \left.+\sum_{j=0}^{k} \sum_{l=0}^{j} X_{2, i}(l) X_{2, i}(j-l) X_{2, i}(k-j)\right) \\
& -\breve{g}_{12}\left(X_{2, i}(\kappa)-X_{3, i}(\kappa)\right) \\
& -\breve{g}_{13}\left(X_{2, i}(\kappa)-X_{3, i}(\kappa)\right) \\
& +\left(\frac{a}{w}\right) \frac{(w)^{k}}{k !} \cos \left(\left(\frac{T}{M}\right) m+\frac{\pi k}{2}\right) \\
& \left.+\frac{0.02(1.1)^{k}}{k !} \sin \left(\left(\frac{T}{M}\right) m+\frac{\pi k}{2}\right)\right), \\
& Y_{2, i}(k+1)=b \Gamma_{\alpha_{4}} X_{2, i}(\kappa) \text {, } \\
& X_{3, i}(k+1) \\
& =\Gamma_{\alpha_{5}}\left(\sum_{l=0}^{k} X_{3, i}(l) X_{3}(k-l)-X_{3, i}(\kappa)-Y_{3, i}(k)\right. \\
& -r\left(-\sum_{l=0}^{k} X_{3, i}(l) X_{3, i}(k-l)\right. \\
& \left.+\sum_{j=0}^{k} \sum_{l=0}^{j} X_{3}(l) X_{3, i}(j-l) X_{3, i}(k-j)\right)
\end{aligned}
$$



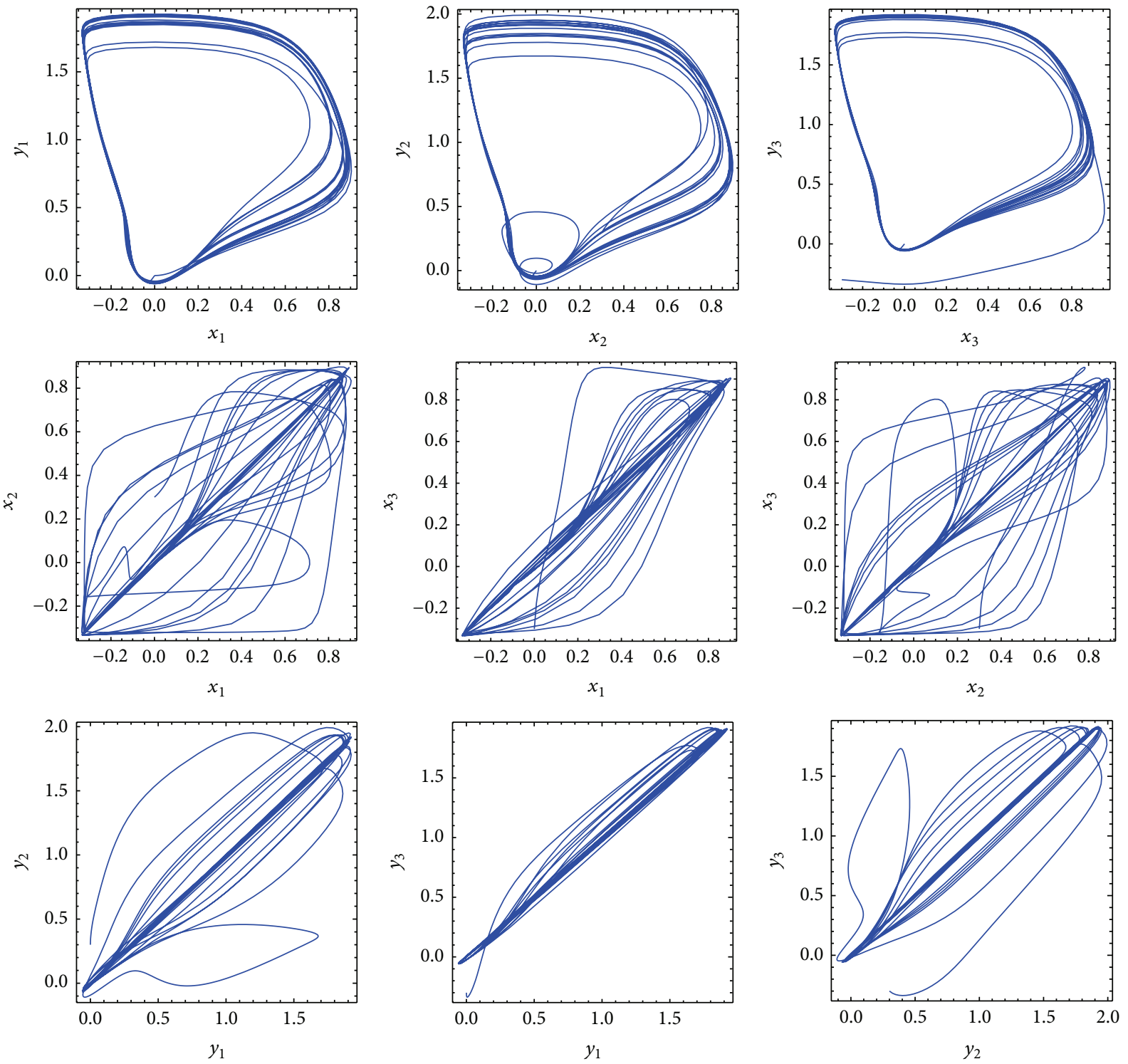

FIGURE 3: Phase plot of chaotic behavior of chaotic FHN neuromsis, with $\alpha_{1}=\alpha_{3}=\alpha_{5}=0.9, \alpha_{2}=\alpha_{4}=\alpha_{6}=0.8$.

$$
\begin{aligned}
& -\breve{g}_{12}\left(X_{3, i}(\kappa)-X_{1, i}(\kappa)\right) \\
& -\breve{g}_{13}\left(X_{3, i}(\kappa)-X_{2, i}(\kappa)\right) \\
& +\left(\frac{a}{w}\right) \frac{(w)^{k}}{k !} \cos \left(\left(\frac{T}{M}\right) m+\frac{\pi k}{2}\right) \\
& \left.+\frac{0.02(1.2)^{k}}{k !} \sin \left(\left(\frac{T}{M}\right) m+\frac{\pi k}{2}\right)\right),
\end{aligned}
$$

$Y_{3, i}(k+1)=b \Gamma_{\alpha_{6}} X_{3, i}(\kappa)$

such that $X_{1, i}(0)=x_{1, i}\left(t_{i-1}\right)=x_{1, i-1}\left(t_{i-1}\right), Y_{1, i}(0)=y_{1, i}\left(t_{i-1}\right)=$ $y_{1, i-1}\left(t_{i-1}\right), X_{2, i}(0)=x_{2, i}\left(t_{i-1}\right)=x_{2, i-1}\left(t_{i-1}\right), Y_{2, i}(0)=$ $y_{2, i}\left(t_{i-1}\right)=y_{2, i-1}\left(t_{i-1}\right), X_{3, i}(0)=x_{3, i}\left(t_{i-1}\right)=x_{3, i-1}\left(t_{i-1}\right)$, and $Y_{3, i}(0)=y_{3, i}\left(t_{i-1}\right)=y_{3, i-1}\left(t_{i-1}\right)$.
Finally, starting with $X_{1,0}(0)=c_{1}, Y_{1,0}(0)=c_{2}, X_{2,0}(0)=$ $c_{3}, Y_{2,0}(0)=c_{4}, X_{3,0}(0)=c_{5}$ and $Y_{3,0}(0)=c_{6}$ and using the recurrence relation given in (14), the multistep solution can be obtained as in (13).

\section{A Test Problem for the Fractional-Order Chaotic FHN Neurons Model}

In this work, we carefully propose the MSGDTM, a reliable modification of the GDTM that improves the convergence of the series solution. The method provides immediate and visible symbolic terms of analytic solutions as well as numerical approximate solutions to both linear and nonlinear differential equations. Moreover, we shall demonstrate the accuracy of the MSGDT scheme against the Mathematica built-in fourth-order Runge-Kutta (RK4) procedure for the solutions of multiple chaotic FHN neurons model in the case of 

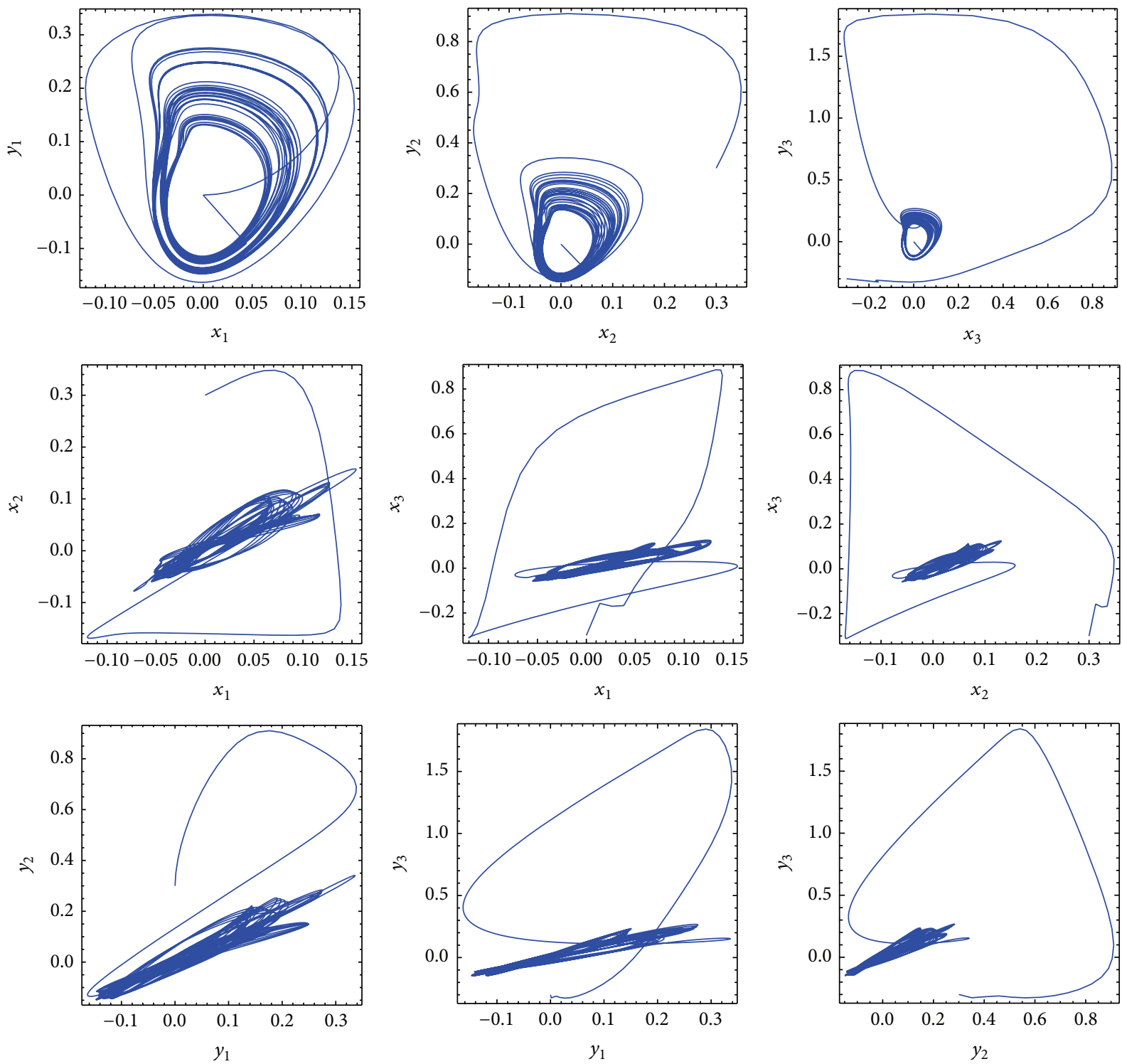

FIGURE 4: Phase plot of chaotic behavior of chaotic FHN neuronsis, with $\alpha_{1}=\alpha_{3}=\alpha_{5}=0.9, \alpha_{2}=\alpha_{4}=\alpha_{6}=0.8$.

integer order derivatives. The MSGDT scheme is coded in the computer algebra package Mathematica. The Mathematica environment variable digits controlling the number of significant digits are set to 20 in all the calculations done in this paper. The time range studied in this work is $[0,250]$ and the step size $\Delta t=0.1$. In this regard, we take the initial condition for chaotic FHN neurons model such as $x_{1}(0)=1$, $y_{1}(0)=0, x_{2}(0)=0.3, y_{2}(0)=0.3, x_{3}(0)=-0.3$, and $y_{3}(0)=-0.3$ with parameters $r=10, b=1$ and $a=0.1$, whilst $g_{12}=0.011, g_{13}=0.012, g_{13}=0.013, \Delta g_{12}=0.1$, $\Delta g_{12}=0.14, \Delta g_{13}=0.18, \breve{g}_{12}=g_{12}+\Delta g_{12}, \breve{g}_{13}=g_{13}+\Delta g_{13}$, and $\breve{g}_{23}=g_{23}+\Delta g_{23}$.

Figure 1 shows the phase portrait for the classical multiple chaotic FHN neurons model, when $\alpha_{1}=\alpha_{2}=\alpha_{3}=\alpha_{4}=\alpha_{5}=$ $\alpha_{6}=1$, using the MSGDT and RK4 methods. However, it can be seen that the results obtained using the MSGDTM match the results of the RK4 method very well, which implies that the MSGDTM can predict the behavior of these variables accurately for the region under consideration. Additionally, Figures 2, 3, and 4 show the phase portrait for the fractional multiple chaotic FHN neurons using the MSGDTM. From the numerical results in Figures 2, 3, and 4, it is clear that the approximate solutions depend continuously on the timefractional derivative $\alpha_{\mathrm{i}}, i=1,2,3,4,5,6$. The effective dimension $\sum$ of (10) is defined as the sum of orders $\alpha_{1}+\alpha_{2}+$ $\alpha_{3}+\alpha_{3}+\alpha_{5}+\alpha_{6}=\sum$. In the meantime, we can see that the chaos exists in the fractional-order multiple chaotic FHN neurons model with order as low as 5.1.

\section{Conclusions}

In this paper, a multistep generalized differential transform method has been successfully applied to find the numerical 
solutions of the fractional-order multiple chaotic FitzHughNagumo neurons model. This method has the advantage of giving an analytical form of the solution within each time interval which is not possible using purely numerical techniques like the fourth-order Runge-Kutta method (RK4). We conclude that MSGDT method is a highly accurate method in solving a broad array of dynamical problems in fractional calculus due to its consistency used in a longer time frame.

The reliability of the method and the reduction in the size of computational domain give this method a wider applicability. Many of the results obtained in this paper can be extended to significantly more general classes of linear and nonlinear differential equations of fractional order.

\section{Conflict of Interests}

The authors declare that there is no conflict of interests regarding the publication of this paper.

\section{Acknowledgment}

The authors would like to express their thanks to the unknown referees for their careful reading and helpful comments.

\section{References}

[1] D. Noble, "Modeling the heart," Physiology, vol. 19, no. 4, pp. 191-197, 2004.

[2] R. FitzHugh, "Impulses and physiological states in theoretical models of nerve membrane," Biophysical Journal, vol. 1, no. 6 , pp. 445-466, 1961.

[3] B. Jobst, "Brain stimulation for surgical epilepsy," Epilepsy Research, vol. 89, no. 1, pp. 154-161, 2010.

[4] M. Aqil, K.-S. Hong, M.-Y. Jeong, and S. S. Ge, "Detection of event-related hemodynamic response to neuroactivation by dynamic modeling of brain activity," NeuroImage, vol. 63, no. 1 , pp. 553-568, 2012.

[5] Y. Rudy and J. R. Silva, "Computational biology in the study of cardiac ion channels and cell electrophysiology," Quarterly Reviews of Biophysics, vol. 39, no. 1, pp. 57-116, 2006.

[6] O. Abu Arqub, A. El-Ajou, S. Momani, and N. Shawagfeh, "Analytical solutions of fuzzy initial value problems by HAM," Applied Mathematics \& Information Sciences, vol. 7, no. 5, pp. 1903-1919, 2013.

[7] M. Al-Smadi, O. Abu Arqub, and S. Momani, "A computational method for two-point boundary value problems of fourth-order mixed integrodifferential equations," Mathematical Problems in Engineering, vol. 2013, Article ID 832074, 10 pages, 2013.

[8] J. Wang, T. Zhang, and B. Deng, "Synchronization of FitzHughNagumo neurons in external electrical stimulation via nonlinear control," Chaos, Solitons \& Fractals, vol. 31, no. 1, pp. 30-38, 2007.

[9] G.-C. Wu, D. Baleanu, and S.-D. Zeng, "Discrete chaos in fractional sine and standard maps," Physics Letters A, vol. 378, no. 5-6, pp. 484-487, 2014.

[10] C. Li and G. Chen, "Chaos in the fractional order Chen system and its control," Chaos, Solitons \& Fractals, vol. 22, no. 3, pp. 549-554, 2004.
[11] G. C. Wu and D. Baleanu, "Chaos synchronization of the discrete fractional logistic map," Signal Processing, vol. 102, pp. 96-99, 2014.

[12] C. Li and G. Chen, "Chaos and hyperchaos in the fractionalorder Rössler equations," Physica A, vol. 341, no. 1-4, pp. 55-61, 2004.

[13] G.-C. Wu and D. Baleanu, "Discrete fractional logistic map and its chaos," Nonlinear Dynamics, vol. 75, no. 1-2, pp. 283-287, 2014.

[14] C. Li and G. Peng, "Chaos in Chen's system with a fractional order," Chaos, Solitons \& Fractals, vol. 22, no. 2, pp. 443-450, 2004.

[15] W. H. Deng and C. P. Li, "Chaos synchronization of the fractional Lü system," Physica A, vol. 353, no. 1-4, pp. 61-72, 2005.

[16] O. Abu Arqub, Z. Abo-Hammour, and S. Momani, "Application of continuous genetic algorithm for nonlinear system of second-order boundary value problems," Applied Mathematics and Information Sciences, vol. 8, no. 1, pp. 253-248, 2014.

[17] J. Rinzel and J. P. Keaner, "Hopf bifurcation to repetitive activity in nerve," SIAM Journal on Applied Mathematics, vol. 43, no. 4, pp. 907-922, 1983.

[18] J. Rinzel and J. B. Keller, "Traveling wave solutions of a nerve conduction equation," Biophysical Journal, vol. 13, no. 12, pp. 1313-1337, 1973.

[19] M. Rehan and K.-S. Hong, "Robust synchronization of delayed chaotic FitzHugh-Nagumo neurons under external electrical stimulation," Computational and Mathematical Methods in Medicine, vol. 2012, Article ID 230980, 11 pages, 2012.

[20] M. Rehan, K.-S. Hong, and M. Aqil, "Synchronization of multiple chaotic FitzHugh-Nagumo neurons with gap junctions under external electrical stimulation," Neurocomputing, vol. 74, no. 17, pp. 3296-3304, 2011.

[21] A. A. Soliman, "Numerical simulation of the FitzHugh-Nagumo equations," Abstract and Applied Analysis, vol. 2012, Article ID 762516, 13 pages, 2012.

[22] R. Artebrant, A. Tveito, and G. T. Lines, "A method for analyzing the stability of the resting state for a model of pacemaker cells surrounded by stable cells," Mathematical Biosciences and Engineering, vol. 7, no. 3, pp. 505-526, 2010.

[23] A. Freihat and S. Momani, "Application of multistep generalized differential transform method for the solutions of the fractional-order Chua's system," Discrete Dynamics in Nature and Society, vol. 2012, Article ID 427393, 12 pages, 2012.

[24] A. Freihat and M. Al-Smadi, "A new reliable algorithm using the generalized differential transform method for the numericanalytic solution of fractional-order Liu chaotic and hyperchaotic systems," Pensee Journal, vol. 75, no. 9, pp. 263-276, 2013.

[25] Z. Odibat and S. Momani, "A generalized differential transform method for linear partial differential equations of fractional order," Applied Mathematics Letters, vol. 21, no. 2, pp. 194-199, 2008.

[26] S. Momani and Z. Odibat, "A novel method for nonlinear fractional partial differential equations: combination of DTM and generalized Taylor's formula," Journal of Computational and Applied Mathematics, vol. 220, no. 1-2, pp. 85-95, 2008.

[27] V. S. Erturk, S. Momani, and Z. Odibat, "Application of generalized differential transform method to multi-order fractional differential equations," Communications in Nonlinear Science and Numerical Simulation, vol. 13, no. 8, pp. 1642-1654, 2008. 
[28] A. Freihat and S. Momani, "Adaptation of differential transform method for the numeric-analytic solution of fractional-order Rössler chaotic and hyperchaotic systems," Abstract and Applied Analysis, vol. 2012, Article ID 934219, 13 pages, 2012.

[29] Z. Odibat, S. Momani, and V. S. Ertürk, "Generalized differential transform method: application to differential equations of fractional order," Applied Mathematics and Computation, vol. 197, no. 2, pp. 467-477, 2008.

[30] K. S. Miller and B. Ross, An Introduction to the Fractional Calculus and Fractional Differential Equations, A Wiley-Interscience Publication, John Wiley \& Sons, New York, NY, USA, 1993.

[31] C. Li and W. Deng, "Remarks on fractional derivatives," Applied Mathematics and Computation, vol. 187, no. 2, pp. 777-784, 2007.

[32] J. Sabatier, O. P. Agrawal, and J. A. Tenreiro Machado, Advances in Fractional Calculus: Theoretical Developments and Applications in Physics and Engineering, Springer, Dordrecht, The Netherlands, 2007. 


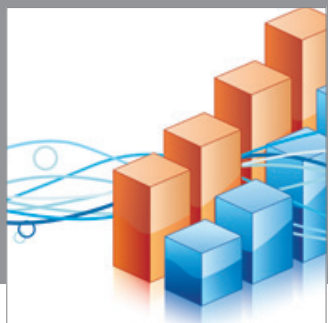

Advances in

Operations Research

mansans

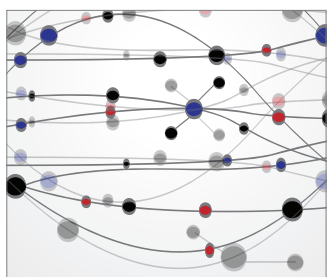

The Scientific World Journal
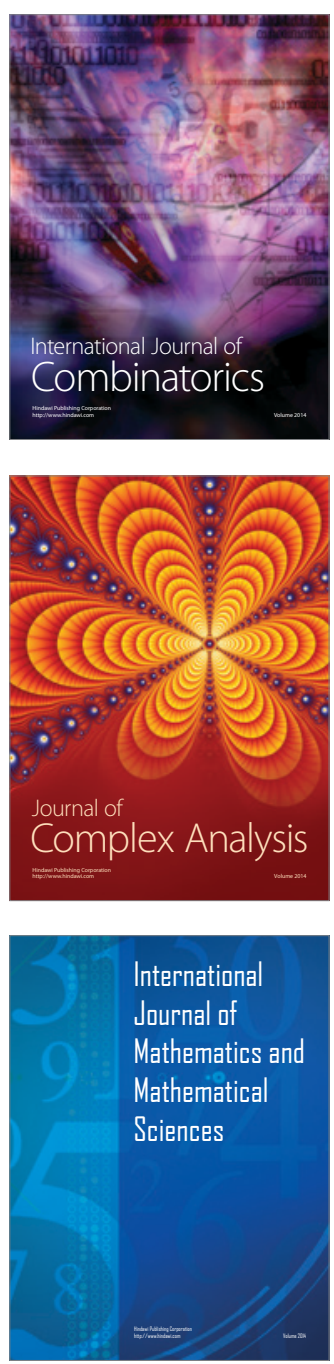
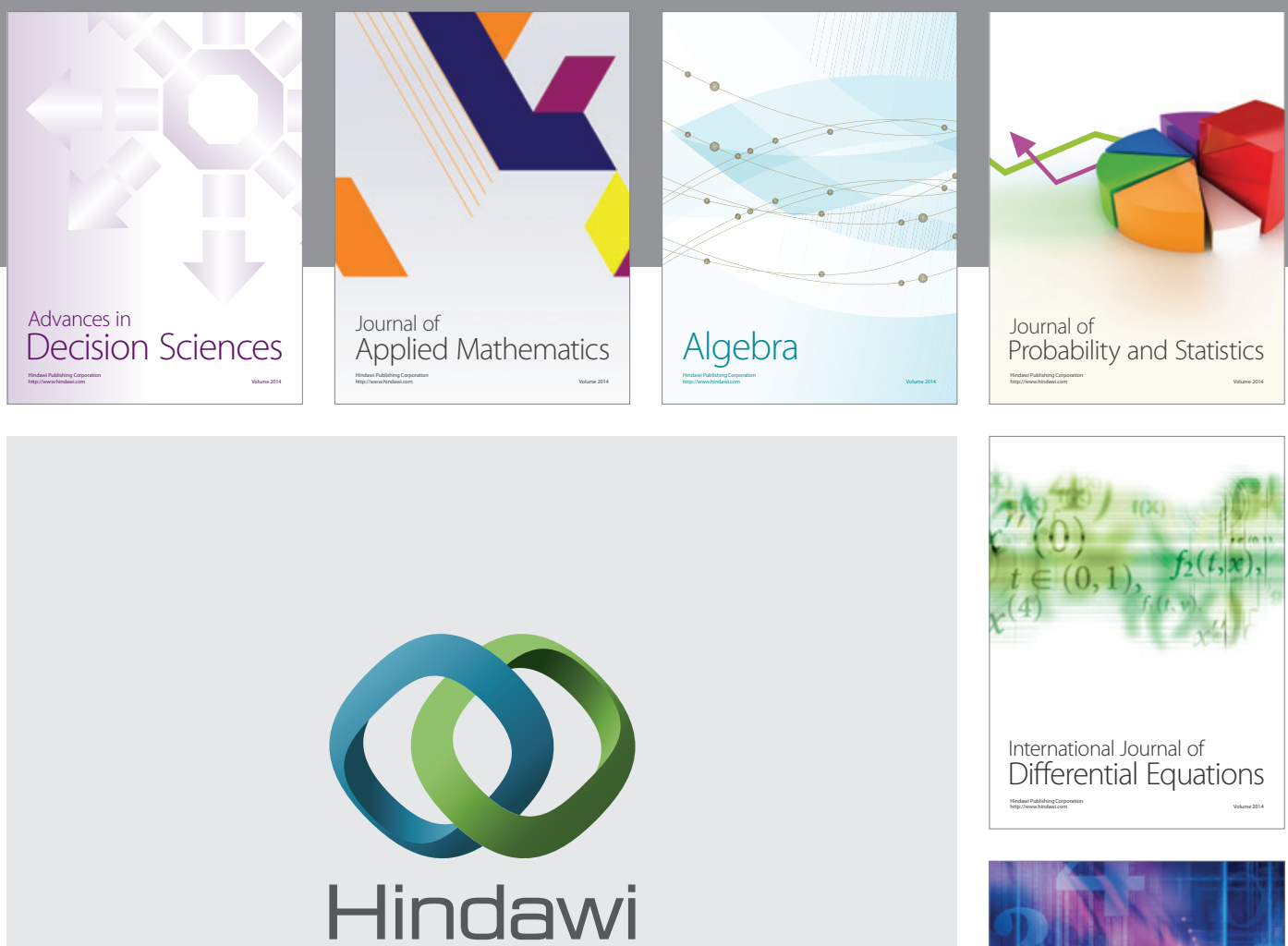

Submit your manuscripts at http://www.hindawi.com
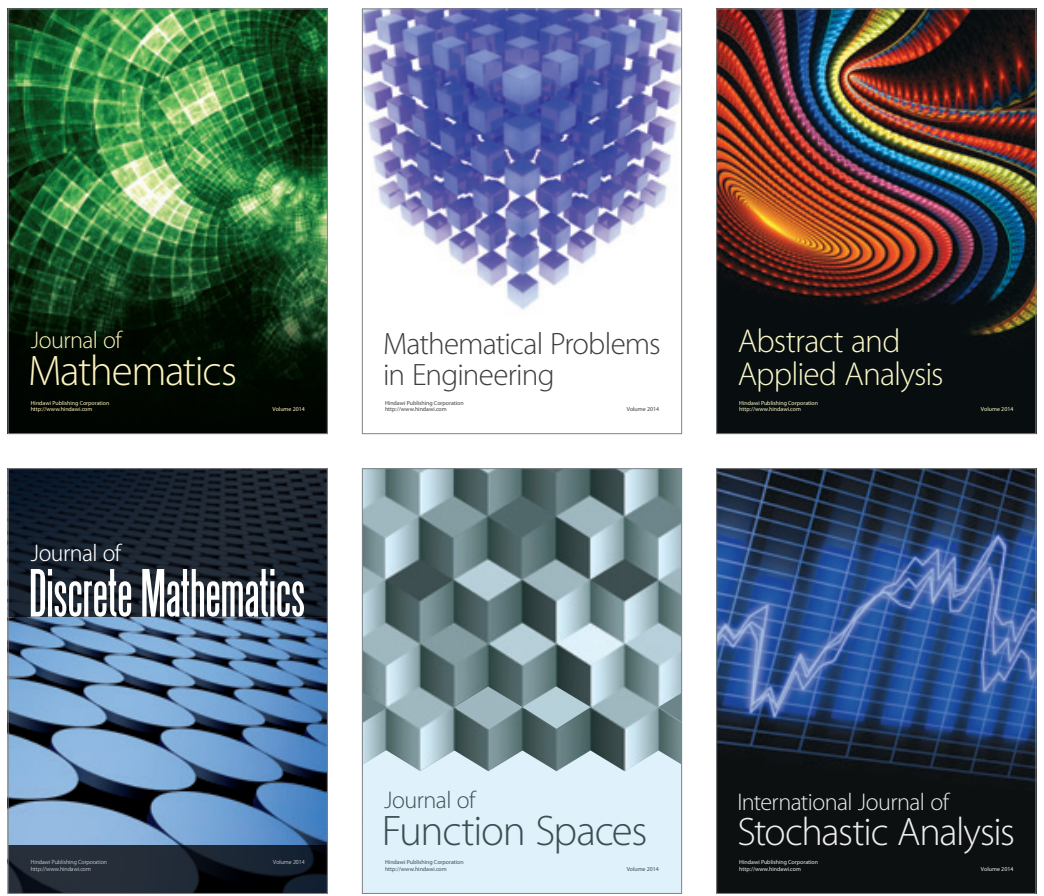

Journal of

Function Spaces

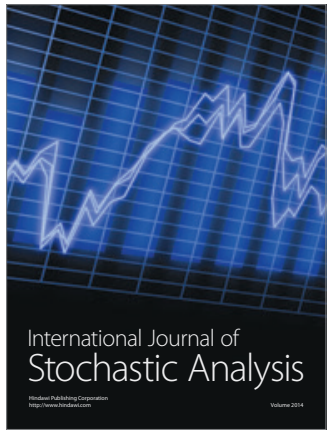

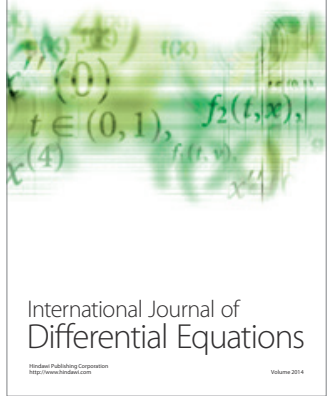
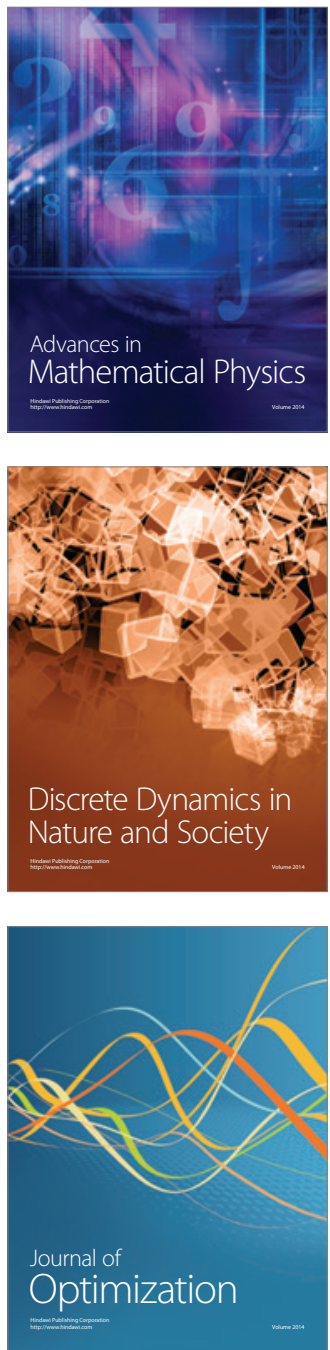